\title{
LOWER BOUNDS ON THE HAUSDORFF MEASURE OF NODAL SETS II
}

\author{
Christopher D. Sogge And Steve Zelditch
}

\begin{abstract}
We give a very short argument showing how the main identity (0.2) from our earlier paper [12] immediately leads to the best lower bound currently known [2] for the Hausdorff measure of nodal sets in dimensions $n \geq 3$.
\end{abstract}

Let $(M, g)$ be a compact smooth Riemannian manifold of dimension $n$ and let $e_{\lambda}$ be real-valued eigenfunction of the associated Laplacian, i.e.,

$$
-\Delta_{g} e_{\lambda}(x)=\lambda^{2} e_{\lambda}(x)
$$

with frequency $\lambda>0$. Recent papers have been concerned with lower bounds for the $(n-1)$-dimensional Hausdorff measure, $\left|Z_{\lambda}\right|$, of the nodal set of $e_{\lambda}$,

$$
Z_{\lambda}=\left\{x \in M: e_{\lambda}(x)=0\right\}
$$

in dimensions $n \geq 3$. When $n=2$ the sharp lower bound by the frequency, $\lambda \lesssim\left|Z_{\lambda}\right|$, was obtained by Brüning in [1] and independently by Yau. For all dimensions, in the analytic case, the sharp upper and lower bounds $\left|Z_{\lambda}\right| \approx \lambda$ were obtained by Donnelly and Fefferman $[4,5]$.

Until recently, the best known lower bound when $n \geq 3$ seems to have been $e^{-c \lambda} \lesssim$ $\left|Z_{\lambda}\right|$ (see [6]). Using a variation (0.2) of an identity of Dong [3], the authors showed in [12] that this can be improved to be $\lambda^{\frac{7}{4}-\frac{3 n}{4}} \lesssim\left|Z_{\lambda}\right|$. Independently Colding and Minicozzi [2] obtained the more favorable lower bound

$$
\lambda^{1-\frac{n-1}{2}} \lesssim\left|Z_{\lambda}\right|
$$

by a different method. Subsequently, the first author and Hezari [7] were also able to obtain the lower bound (0.1) by an argument which was in the spirit of [12]. The purpose of this sequel to [12] is to show that the lower bound (0.1) can also be derived by a very small modification (indeed a simplification) of the original argument of [12].

The lower bounds of $[7,12]$ are based on the identity

$$
\lambda^{2} \int_{M}\left|e_{\lambda}\right| d V=2 \int_{Z_{\lambda}}\left|\nabla_{g} e_{\lambda}\right|_{g} d S
$$

from [12] and the (sharp) lower bound for $L^{1}$-norms

$$
\lambda^{-\frac{n-1}{4}} \lesssim \int_{M}\left|e_{\lambda}\right| d V
$$

which was also established in [12]. Here, $d V$ is the volume element of $(M, g)$.

The lower bound (0.1) is a very simple consequence of the identity $(0.2)$ and the following lemma (which was implicit in [12]).

Received by the editors December 23, 2012. 
Lemma 1. If $\lambda>0$ then

$$
\left\|\nabla_{g} e_{\lambda}\right\|_{L^{\infty}(M)} \lesssim \lambda^{1+\frac{n-1}{2}}\left\|e_{\lambda}\right\|_{L^{1}(M)} .
$$

Indeed if we use (0.2) and then apply Lemma 1, we obtain

$$
\begin{aligned}
\lambda^{2} \int_{M}\left|e_{\lambda}\right| d V=2 \int_{Z_{\lambda}}\left|\nabla_{g} e_{\lambda}\right|_{g} d S & \leq 2\left|Z_{\lambda}\right|\left\|\nabla_{g} e_{\lambda}\right\|_{L^{\infty}(M)} \\
& \lesssim 2\left|Z_{\lambda}\right| \lambda^{1+\frac{n-1}{2}}\left\|e_{\lambda}\right\|_{L^{1}(M)},
\end{aligned}
$$

which of course implies (0.1).

Lemma 1 improves the upper bound on the integral given in Lemma 1 of [12], and its proof is almost the same as the proof of (0.3) in Proposition 2 of [12]:

Proof. For $\rho \in C_{0}^{\infty}(\mathbb{R})$, we define the $\lambda$-dependent family of operators

$$
\chi_{\lambda} f=\int_{-\infty}^{\infty} \rho(t) e^{-i t \lambda} e^{i t \sqrt{-\Delta_{g}}} f d t=\hat{\rho}\left(\lambda-\sqrt{-\Delta_{g}}\right) f=\sum_{j=0}^{\infty} \hat{\rho}\left(\lambda-\lambda_{j}\right) E_{j} f,
$$

on $L^{2}(M, d V)$ with $E_{j} f$ denoting the projection of $f$ onto the $j$ th eigenspace of $\sqrt{-\Delta_{g}}$. Here $0=\lambda_{0}<\lambda_{1} \leq \lambda_{2} \leq \cdots$ are its eigenvalues, and if $\left\{e_{j}\right\}_{j=0}^{\infty}$ is the associated orthonormal basis of eigenfunctions (i.e. $\sqrt{-\Delta_{g}} e_{j}=\lambda_{j} e_{j}$ ), then

$$
E_{j} f=\left(\int_{M} f \overline{e_{j}} d V\right) e_{j} .
$$

We denote the kernel of $\chi_{\lambda}$ by $K_{\lambda}(x, y)$, i.e.,

$$
\chi_{\lambda} f(x)=\int_{M} K_{\lambda}(x, y) f(y) d V(y), \quad(f \in C(M)) .
$$

If the Fourier transform of $\rho$ satisfies $\hat{\rho}(0)=1$, then $\chi_{\lambda} e_{\lambda}=e_{\lambda}$, or equivalently

$$
\int_{M} K_{\lambda}(x, y) e_{\lambda}(y) d V(y)=e_{\lambda}(x)
$$

Thus, $K_{\lambda}$ is a reproducing kernel for $e_{\lambda}$ if $\hat{\rho}(0)=1$.

As in Section 5.1 in [10], we choose $\rho$ so that the reproducing kernel $K_{\lambda}(x, y)$ is uniformly bounded by $\lambda^{\frac{n-1}{2}}$ on the diagonal as $\lambda \rightarrow+\infty$. This is essential for the proof of (0.4). If we assume that $\rho(t)=0$ for $|t| \notin[\varepsilon / 2, \varepsilon]$, with $\varepsilon>0$ being a fixed number which is smaller than the injectivity radius of $(M, g)$, then it is proved in Lemma 5.1.3 of [10] that

$$
K_{\lambda}(x, y)=\lambda^{\frac{n-1}{2}} a_{\lambda}(x, y) e^{i \lambda r(x, y)},
$$

where $a_{\lambda}(x, y)$ is bounded with bounded derivatives in $(x, y)$ and where $r(x, y)$ is the Riemannian distance between points. This WKB formula for $K_{\lambda}(x, y)$ is known as a parametrix and may be obtained from the Hörmander parametrix for $e^{i t \sqrt{-\Delta}}$ in [8] or from the Hadamard parametrix for $\cos t \sqrt{-\Delta}$. We refer to $[10,11]$ for the background.

It follows from (0.7) that

$$
\left|\nabla_{g} K_{\lambda}(x, y)\right| \leq C \lambda^{1+\frac{n-1}{2}}
$$


and therefore,

$$
\begin{aligned}
\sup _{x \in M}\left|\nabla_{g} \chi_{\lambda} f(x)\right| & =\sup _{x}\left|\int f(y) \nabla_{g} K_{\lambda}(x, y) d V\right| \\
& \leq\left\|\nabla_{g} K_{\lambda}(x, y)\right\|_{L^{\infty}(M \times M)}\|f\|_{L^{1}} \\
& \leq C \lambda^{1+\frac{n-1}{2}}\|f\|_{L^{1}} .
\end{aligned}
$$

To complete the proof of the Lemma, we set $f=e_{\lambda}$ and use that $\chi_{\lambda} e_{\lambda}=e_{\lambda}$.

We note that $K_{\lambda}(x, y)$ has quite a different structure from the kernels of the spectral projection operators $E_{[\lambda, \lambda+1]}=\sum_{j: \lambda_{j} \in[\lambda, \lambda+1]} E_{j}$ and the estimate in Lemma 1 is quite different from the sup norm estimate in Lemma 4.2.4 of [10]. Indeed, in a $\lambda^{-1}$ neighborhood of the diagonal, the spectral projections kernel $E_{[\lambda, \lambda+1]}(x, y)$ is of size $\lambda^{n-1}$. For instance, in the case of the standard sphere $S^{n}$, the kernel of the orthogonal projection $E_{k}$ onto the space of spherical harmonics of degree $k \simeq \lambda$ is the constant $E_{k}(x, x)=\frac{\lambda^{n-1}}{V o l\left(S^{n}\right)}$ on the diagonal. We are able to choose the test function $\rho$ above, so that the reproducing kernel $K_{\lambda}(x, y)$ is uniformly of size $\lambda^{\frac{n-1}{2}}$ (as in [9] and [10] Section 5.1) because we only need it to reproduce eigenfunctions $e_{\lambda}$ of one eigenvalue and because it does not matter how $K_{\lambda}$ acts on eigenfunctions of other eigenvalues. From the viewpoint of Lagrangian distributions, the Lagrangian manifold $\Lambda_{x}$ associated to both $E_{[\lambda, \lambda+1]}(x, y)$ and $K_{\lambda}(x, y)$ for fixed $x$ is the flowout $\Lambda_{x}=\bigcup_{t \in \operatorname{supp} \rho} G^{t} S_{x}^{*} M \subset S^{*} M$ of the unit-cosphere $S_{x}^{*} M$ under the geodesic flow $G^{t}$. The natural projection of $\Lambda_{x}$ to $M$ has a large singularity along $S_{x}^{*} M$ which causes the $\lambda^{n-1}$ blowup of $E_{[\lambda, \lambda+1]}(x, y)$ at $y=x$, but the projection is a covering map for the part of $\Lambda_{x}$ where $t \in[\varepsilon, 2 \varepsilon]=\operatorname{supp} \rho$. The parametrix (0.7) reflects the fact that the test function $\rho$ cuts out all of $\Lambda_{x}$ except where its projection to $M$ is a covering map. For further discussion of the geometry underlying Lagrangian distributions we refer to $[10,11,13]$.

Finally, we briefly compare the proof of $(0.1)$ in this note with the estimates in [12]:

- Instead of Lemma 0.1 , the estimate $\left\|\nabla_{g} e\right\|_{L^{\infty}(M)} \lesssim \lambda^{1+\frac{n-1}{2}}\left\|e_{\lambda}\right\|_{L^{2}}$ was used in [12]. The latter estimate is a consequence of the pointwise local Weyl law for $\left|\nabla e_{\lambda}(x)\right|^{2}$.

- In [12] the authors proved the lower bounds (0.3) by showing that

$$
\left\|e_{\lambda}\right\|_{L^{\infty}(M)} \lesssim \lambda^{\frac{n-1}{2}}\left\|e_{\lambda}\right\|_{L^{1}(M)},
$$

by essentially the same argument as in Lemma 1 . In the proof given in this note, (0.3) is not used in the proof of (0.1) since the factor $\left\|e_{\lambda}\right\|_{L^{1}(M)}$ cancels out in the left and right sides.

\section{References}

[1] J. Brüning, Über Knoten Eigenfunktionen des Laplace-Beltrami Operators, Math. Z. 158 (1978), 15-21.

[2] T.H. Colding and W. P. Minicozzi II, Lower bounds for nodal sets of eigenfunctions, Comm. Math. Phys. 306 (2011), 777-784.

[3] R.T. Dong, Nodal sets of eigenfunctions on Riemann surfaces, J. Different. Geom. 36 (1992), 493-506. 
[4] H. Donnelly and C. Fefferman, Nodal sets of eigenfunctions on Riemannian manifolds, Invent. Math. 93 (1988), 161-183.

[5] H. Donnelly and C. Fefferman, Nodal sets for eigenfunctions of the Laplacian on surfaces, J. Amer. Math. Soc. 3 (1990), 333-353.

[6] Q. Han and F.H. Lin, Nodal sets of solutions of Elliptic Differential Equations, book in preparation (online at http://www.nd.edu/qhan/nodal.pdf).

[7] H. Hezari and C.D. Sogge, A natural lower bound for the size of nodal sets, Anal. PDE 5 (2012), no. 5, 1133-1137. arXiv:1107.3440.

[8] L. Hörmander, The spectral function of an elliptic operator, Acta Math., 121 (1968), 193-218.

[9] C.D. Sogge, Concerning the $L^{p}$ norm of spectral clusters for second-order elliptic operators on compact manifolds, J. Funct. Anal. 77 (1988), 123-138.

[10] C.D. Sogge, Fourier integrals in classical analysis, Cambridge Tracts in Mathematics, 105, Cambridge University Press, Cambridge, 1993.

[11] C.D. Sogge, Hangzhou lectures on eigenfunctions of the Laplacian, Annals of Mathematics Studies, Princeton University Press, to appear.

[12] C.D. Sogge and S. Zelditch, Lower bounds on the Hausdorff measure of nodal sets, Math. Res. Lett. 18 (2011), 25-37.

[13] S. Zelditch, CBMS Lectures, in preparation.

Department of Mathematics, Johns Hopkins University, Baltimore, MD, USA

E-mail address: csogge@math.jhu.edu

Department of Mathematics, Northwestern University, Evanston, IL, USA

E-mail address: zelditch@math.northwestern.edu 